\title{
The XEN of reprogramming
}

Cell Research (2016) 26:147-148. doi:10.1038/cr.2016.9; published online 22 January 2016

\begin{abstract}
Reprogramming to pluripotency has thus far required complex procedures involving nuclear transfer, cell fusion or genetic manipulation. Two recent papers from Hongkui Deng's group now show various cell types can be reprogrammed simply by chemicals through an extraembryonic endoderm-like phase instead of the primitive streak-like mesendoderm induced by Yamanaka factors.
\end{abstract}

Pluripotent stem cells (PSCs) can proliferate indefinitely in culture, and differentiate into all cell types of the adult under proper cues [1]. Hence, they represent an unlimited source for the generation of the cells that are scarce or inaccessible in the adult. Their use has been influential in the generation of genetically modified animal models as well as modeling disease and development in a dish. Unlike plants and phylogenetically lower metazoans such as planaria or hydra, PSCs have been unidentified in adult mammals. They only occur as transitory intermediates during the mammalian ontogeny and undergo sequential differentiation to form the entire organism. Nevertheless, somatic cells can be induced to acquire pluripotency by a variety of approaches [2]. One of the promising uses of these induced PSCs (iPSCs) is the generation of functional cells for replacement therapies since cells derived from autologous iPSCs will not, in theory, invoke immunological rejection upon transplantation. Thus, iPSCs constitute a revolutionary tool towards the advancement of personalized medicine [3].

The first demonstration of reprogramming was almost 50 years ago when adult frogs were cloned from enucleated eggs transferred with somat- ic nuclei. These experiments indicated that cytoplasmic factors are sufficient to reprogram the somatic nuclei. Establishment of conditions to culture PSCs isolated from human embryos allowed for the reprogramming of the human somatic nuclei to pluripotent state by fusing the somatic cells with PSCs. In 2006, concurrent expression of 4 transcription factors, OCT4, SOX2, KLF4 and c-MYC (Yamanaka factors), was found to be sufficient to induce pluripotency [4]. Although the past decade has seen methodological improvements, generation of iPSCs still requires genetic manipulation that hinders their therapeutic application. The chemical cocktail developed by Zhao et al. [5] of Deng's group circumvents this problem.

The findings of Deng's group are actually an extension of their work in 2011, when they embarked to first identify the chemicals that can replace SOX2, KLF4 and c-MYC (SKM) in Yamanaka factors-based reprogramming [6]. Toward this end, they used mouse embryonic fibroblasts (MEFs) that express GFP under the control of Oct4 transcriptional elements (OG) as a reporter system to indicate pluripotency. By candidate approaches and chemical screening, they identified VPA, CHIR99021, 616452 and tranylcypromine (VC6T) to be sufficient to confer pluripotency to fibroblasts when co-administered with OCT4-expressing lentivirus [6]. In their next report in 2013, they looked for chemical substitutes for OCT4 by screening OG MEFs transduced with lentiviruses expressing SKM [7]. They identified Forskolin and DZNep as the most potent factors. Combining them with VC6T (VC6TFZ) proved to be sufficient for the induction of iPSCs from fibroblasts without the use of transgenes. Forskolin promoted the mesenchymal-to-epithelial transition of the fibroblasts, which is the first phase in their conversion to iPSCs, while DZNep inclusion in the second step was necessary for the conversion of the intermediate epitheloid cells to iPSCs that they termed chemically induced PSCs (CiPSCs). Additionally, they generated CiPSCs from adult fibroblasts and adipose-derived stem cells. Phenotypic characterization showed that CiPSCs have de-repressed epigenetic state of the pluripotency factors and similar gene expression profile to embryonic stem cells. Importantly, they can form teratoma and chimera with germline transmission, the golden standards of pluripotency. Characterization of the molecular dynamics by gene expression analysis showed that extraembryonic endoderm (XEN) markers Sall4, Gata4, Gata6 and Sox 17 are upregulated during the early stages of chemical-induced reprogramming, indicating that a XEN-like intermediate state is induced before pluripotency is reached.

The study published by Zhao et al. [5] now confirms that CiPSCs are derived via a XEN-like state, which allowed them to enhance the efficiency of the chemical-induced reprogramming by dissection of the cell fate transitions. They first observed that CiPSCs emerged from epitheloid colonies that expressed XEN markers and knocking these markers down hampered CiPSC formation. These epitheloid colonies were similar to embryo-derived XEN in their gene expression profile, developmental and reprogramming potentials. This information allowed 
them to screen additional chemicals that boost the induction of XEN-like colonies from fibroblasts in short term. They identified that EPZ004777 (E) and AM580 (A) enhanced the formation of XEN-like colonies by $>50$-fold when used with the original VC6TF cocktail at an increased concentration of CHIR99021. Next they screened chemicals that enhanced the XEN-toCiPSC transition and found that 5-aza$\mathrm{dC}$ (D) synergistically enhanced the effect of VC6TFAZ with EPZ004777 (E) or SGC0946, particularly when an optimized PSC medium was used at the last phase of the reprogramming. Optimizing the duration and dosage of the treatments shortened the reprogramming and increased the efficiency 500-1 000 -fold than the original protocol. The optimized protocol generates $1000-9$ 000 CiPSC colonies from 50000 MEFs.

Another report from Deng's group by Ye et al. [8] now shows that the core components of this chemical cocktail is applicable to the reprogramming of neural stem cells and intestinal epithelial cells, whereby it enlarges the spectrum of the somatic cell source from mesoderm-derived fibroblasts to ectoderm and endoderm lineages, respectively. They first performed lineage tracing experiments via fibroblast specific protein 1 (Fsp1) to confirm that CiPSCs can be indeed generated from differentiated fibroblasts rather than a specific cell type amenable to pluripotency within the culture. Neural stem cells isolated from mouse brains by SSEA1 surface marker required another chemical, Ch55 (5), in addition to VC6TFE and decreased dosage of 616452 (6). They then tried to reprogram epithelial cells isolated from the small intestine based on the markers, EpCAM or VILLIN. Again, the core chemical cocktail VC6TF together with AM580 during the first phase and with DZNep during the second phase was sufficient, albeit this time a higher dose of 616452 was required during the first phase. Gene expression analysis showed that all these cells were reprogrammed through a XEN-like intermediate. Thus, the core factors VC6TF is applicable to multiple cell types, all of which transit through a XEN-like state, but efficient reprogramming may require optimization and additional boosters depending on the cell type.

A significant observation of Deng's group is that reprogramming by the chemical cocktail follows a different molecular roadmap from reprogramming by Yamanaka factors. Indeed, knocking down XEN-related genes did not hamper reprogramming by Yamanaka factors unlike the chemical-induced reprogramming. Yamanaka factors first induce cell-specific developmental gene expression patterns $[9,10]$ and primitive streak mesendoderm genes [11] before pluripotency is reached. This is the reverse of ontogeny, where the pluripotent epiblast gives rise to primitive streak mesendoderm, which in turn forms mesoderm- and endoderm-derived cells. In this aspect, Yamanaka factors induce the cells to go backward in ontogeny. $\mathrm{XEN}$, on the other hand, mostly generates the extraembryonic tissue, yolk sac. Therefore, somatic cells in the adult are not even its developmental descents. However, although the developmental fates of epiblast and XEN are divergent from each other in vivo, XEN cells have been derived from PSCs in vitro [12]. These observations combined with the observation of XEN-to-PSC transition by Zhao et al. [5] indicate that cells can be more plastic in vitro, and the chemical cocktail of Deng's group might be hijacking such plasticity of XEN cells instead of reversing ontogeny. It is thus possible that the same principle can be applied to other embryonic sources, such as neural crest cells, to achieve pluripotency by hijacking their plasticity as an intermediate state to pluripotency in vitro.

The chemical induction strategy provides the convenience of a controllable platform for reprogramming by modifying the dosage, duration and combination of the inductive factors.
Additionally, it appears safe since it does not involve genomic integration events. However, it is important to evaluate the mutagenic potential and irreversible toxicity of the chemicals. Another limiting factor is the long duration. CiPSC formation from fibroblasts takes 40 days even with the optimized protocol of Zhao et al. [5] versus 2-3 weeks by Yamanaka factors. Additional optimization of the protocol and/or chemical cocktail is expected to shorten the duration in future. It is also of essence for the regenerative medicine field to assess whether a similar chemical cocktail can be used to reprogram human cells. The observations by Deng's group show us that the same destination can be reached by multiple routes in vitro and not necessarily by mimicking the currently known developmental rules.

\section{Ergin Beyret ${ }^{1}$,}

Juan Carlos Izpisua Belmonte ${ }^{1}$

${ }^{I}$ Gene Expression Laboratory, The Salk Institute for Biological Studies, 10010 North Torrey Pines Road, La Jolla, CA 92037, USA

Correspondence: Juan Carlos Izpisua Belmonte E-mail: belmonte@salk.edu

Tel: +1-858-453-4100 ext. 1130

\section{References}

1 Wu J, Okamura D, Li M, et al. Nature 2015; 521:316-321.

2 Yamanaka S, Blau HM. Nature 2010; 465:704-712.

3 Wu J, Izpisua Belmonte JC. Cell Stem Cell 2015; 17:509-525.

4 Takahashi K, Yamanaka S. Cell 2006; 126:663-676.

5 Zhao Y, Zhao T, Guan J, et al. Cell 2015; 163:1678-1691.

6 Li Y, Zhang Q, Yin X, et al. Cell Res 2011; 21:196-204.

7 Hou P, Li Y, Zhang X, et al. Science 2013; 341:651-654.

8 Ye J, Ge J, Zhang X, et al. Cell Res 2016; 26:34-45.

9 Polo JM, Anderssen E, Walsh RM, et al. Cell 2012; 151:1617-1632.

10 Ohnishi K, Semi K, Yamamoto T, et al. Cell 2014; 156:663-677.

11 Takahashi K, Tanabe K, Ohnuki M, et al. Nat Commun 2014; 5:3678.

12 Cho LT, Wamaitha SE, Tsai IJ, et al. Development 2012; 139:2866-2877. 